\title{
SIMULTANEOUS DETERMINATION OF HYDROCHLOROTHIAZIDE AND TELMISARTAN FROM PHARMACEUTICAL PREPARATIONS USING CAPILLARY ELECTROPHORESIS
}

\author{
ȘTEFANA STĂCESCUa, GABRIEL HANCU ${ }^{a *}$, LASZLO GAGYI \\ RUXANDRA MARIA SOARE ${ }^{a}$, HAJNAL KELEMEN ${ }^{a}$
}

\begin{abstract}
Fixed-dose combinations of an angiotensin II receptor antagonist (telmisartan) and a thiazide diuretic (hydrochlorothiazide) provide an effective antihypertensive therapy while promoting patient compliance with the convenience of once-daily single dose administration. The current study investigated two capillary electrophoretic methods: a capillary zone electrophoresis (CZE) and micellar electrokinetic capillary chromatography one (MEKC) for the simultaneous determination of hydrochlorothiazide and telmisartan. In order to optimize the analytical conditions, efforts were focused on establishing the influence of several experimental parameters on the separation: buffer composition, concentration and $\mathrm{pH}$, applied voltage, temperature, injection pressure and time. The separation of the two analytes can be achieved by CZE using a $25 \mathrm{mM}$ phosphate buffer at $\mathrm{pH} 2.50$ and by MEKC using a $50 \mathrm{mM}$ borate buffer at $\mathrm{pH} 9.50$ containing $25 \mathrm{mM}$ sodium dodecyl sulfate as surfactant. The analytical performance of the two methods was verified in terms of their linearity, precision, accuracy and robustness, and the methods were applied for the determination of the analytes from fixed-dose combinations.
\end{abstract}

Keywords: hydrochlorothiazide, telmisartan, fixed dose combinations, capillary electrophoresis, pharmaceutical preparations

\footnotetext{
a University of Medicine and Pharmacy Tîrgu Mures, Faculty of Pharmacy, Department of Pharmaceutical Chemistry,38 Gh. Marinescu str., RO-540139, Tîrgu Mureș, Romania.

b SC Vim Spectrum SRL Tîrgu Mureș, 409 Corunca, RO-547367, Corunca, Romania.

*Corresponding author: gabriel.hancu@umftgm.ro
} 


\section{INTRODUCTION}

Hypertension is the primary cause for morbidity and mortality caused by cardiovascular diseases all over the world. The goal of antihypertensive therapy is to abolish the risks associated with high blood pressure (BP) without adversely affecting the quality of life [1].

Obtaining the target BP level by monotherapy can be challenging currently, especially for the patients with associated comorbidities; as it is proven that the majority of hypertensive patients need two or more antihypertensive drugs to lower their BP effectively [2,3].

Angiotensin II receptor antagonists (ARAs) and thiazide diuretics have a well-established place in the management of hypertension, exhibiting complementary modes of action. Fixed-dose combinations of an ARA and hydrochlorothiazide provide a convenient and effective treatment option for patients who do not achieve BP targets on monotherapy. One such fixed-dose combination is the one represented by telmisartan and hydrochlorothiazide [4].

Hydrochlorothiazide (HCT) (6-chloro-1,1-dioxo-3,4-dihydro-2H-1,2,4benzothiadiazine-7-sulfonamide) is a thiazide diuretic that increases the rate of urine excretion by the kidney, primarily through decreased tubular reabsorption of sodium and chloride, and also by increased osmotic transport of water to the renal tubules [5].

Telmisartan (TEL) (2-(4-\{[4-Methyl-6-(1-methyl-1H-1,3-benzodiazol-2yl)-2-propyl-1H-1,3-benzodiazol-1-yl]methyl\}phenyl)benzoic acid) is a ARA that shows high affinity for the angiotensin II receptor type 1 , which is responsible for vasoconstriction, being used in the management of hypertension [5].

The structural characteristics of the two analytes are presented in

\section{Figure 1.}

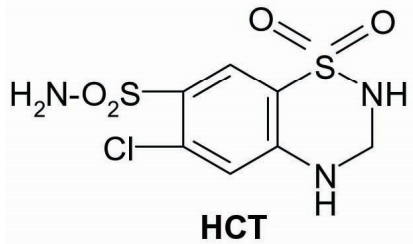

HCT

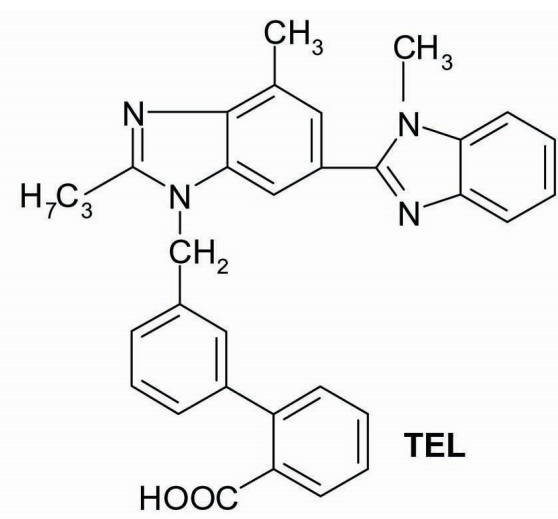

Figure 1. Chemical structures of HCT and TEL 
Taking into consideration the great prevalence and importance of this type of fixed dose combination in modern therapy, the elaboration of new methods of analysis for the simultaneous determination of combined substances can be considered a necessity and also a challenge for the analysts.

Capillary electrophoresis (CE) is a modern separation technique, considered to be an alternative and also a complementary method in drug analysis to the more frequently used high performance liquid chromatography (HPLC) techniques. In CE, separation is based on the differences in the electrophoretic mobilities of the charged molecules due their charge, size, shape or nature of the background electrolyte (BGE). BGE may contain additives, which can interact with the analytes and modify their electrophoretic mobilities. CE encompasses several capillary electro migration techniques, but among them capillary zone electrophoresis (CZE) and micellar electrokinetic chromatograpgy (MEKC) are the most frequently used in drug analysis [6].

Simultaneous determination of the TEL - HCT combination can be obtained using first-derivative and ratio derivative spectrophotometry [7], UV-spectrophotometry using simultaneous equation method [8,9],TLCdensitometry [7], spectrofluorimetry [7], HPLC [10] and RP-HPLC [11,12,13].

Only one CE method has been published so far for the simultaneous estimation of TEL among other five ARA derivates and HCT from combined pharmaceutical forms [14].

Our aim was the development of simple, efficient and rapid CE protocols for the simultaneous determination of TEL and HCT, the optimization of the analytical conditions and to verify the applicability of the newly developed methods for the determination of the two analytes from fixed dose combination product.

\section{RESULTS AND DISCUSSION}

\section{Preliminary analysis}

The separation in CE is highly dependent on the $\mathrm{pH}$ of the BGE which influences the dissociation of the acidic groups or the protonation of basic functional groups on the analyte. The $\mathrm{pH}$ of the BGE will determine the charge of the compounds and also the magnitude of the electroosmotic flow (EOF).

Several preliminary experiments were made using phosphate BGEs with different compositions at different $\mathrm{pH}$ levels, over a $\mathrm{pH}$ ranging from 2.00 to 11.00 . 
TEL is a weak acid has with pKa values of $3.50,4.10$ and 6.00 , being ionized can be determined over the entire studied $\mathrm{pH}$ range $[15,16]$. HCT is a basic compound with pKa values of 7.90 and 9.20 , and subsequently, will be ionized only in an acidic environment [16].

Both substances can be determined simultaneously by ECZ, over a $\mathrm{pH}$ range between 2.50 and 4.50 , but the best results were obtained using a $\mathrm{pH}$ of 2.50; at this $\mathrm{pH}$, the best peak shapes and the shortest analysis time were obtained.

In MEKC, an anionic surfactant, sodium dodecyl sulfate (SDS) was added in a borate buffer in order to modify the electrophoretic behavior of the analytes; both analytes can be determined by MEKC over a $\mathrm{pH}$ range between 9.00 and 11.00, the best results were obtained at a pH of 9.50 .

\section{Optimization of the analytical conditions}

The concentration of the BGEs was varied from 25 to $100 \mathrm{mM}$; when the concentration of the BGE increased the selectivity of the separation improved; but also migration times increased and higher currents were generated.

In the MEKC method the effects of SDS concentrations (25-50 mM) were investigated; an increase in the SDS concentration may result in a more efficient separation but at the same time, raises the current in the capillary and increases migration times.

In order to determine the optimal voltage to be applied, the influence of voltage $(15-30 \mathrm{kV})$ on the migration times was investigated in the optimized BGE conditions; higher voltages reduced migration times but also increased the current intensity.

The influence of capillary temperature $\left(15-25^{\circ} \mathrm{C}\right)$ was evaluated under the optimized BGE conditions; when temperature increased, migration time decreased.

To determine the optimal injection parameters, the influence of injection time ( $1-5$ seconds) and injection pressure $(30-50$ mbar) were studied in order to attain low detection limits without affecting the quality of peak shape, reproducibility and resolution.

From the above experiments, the optimal separation conditions for the simultaneous analysis of TEL and HCT by ECZ were: $50 \mathrm{mM}$ phosphate buffer, $\mathrm{pH} 2.50$, applied voltage $+25 \mathrm{kV}$, capillary temperature $25^{\circ} \mathrm{C}$, injection parameters $50 \mathrm{mbar} / 1 \mathrm{sec}$., UV detection wavelength $230 \mathrm{~nm}$. Using optimized conditions, we obtained the separation of the two analytes, in approximately 3 minutes, the order of migration being: TEL followed by HCT (Figure 2). 


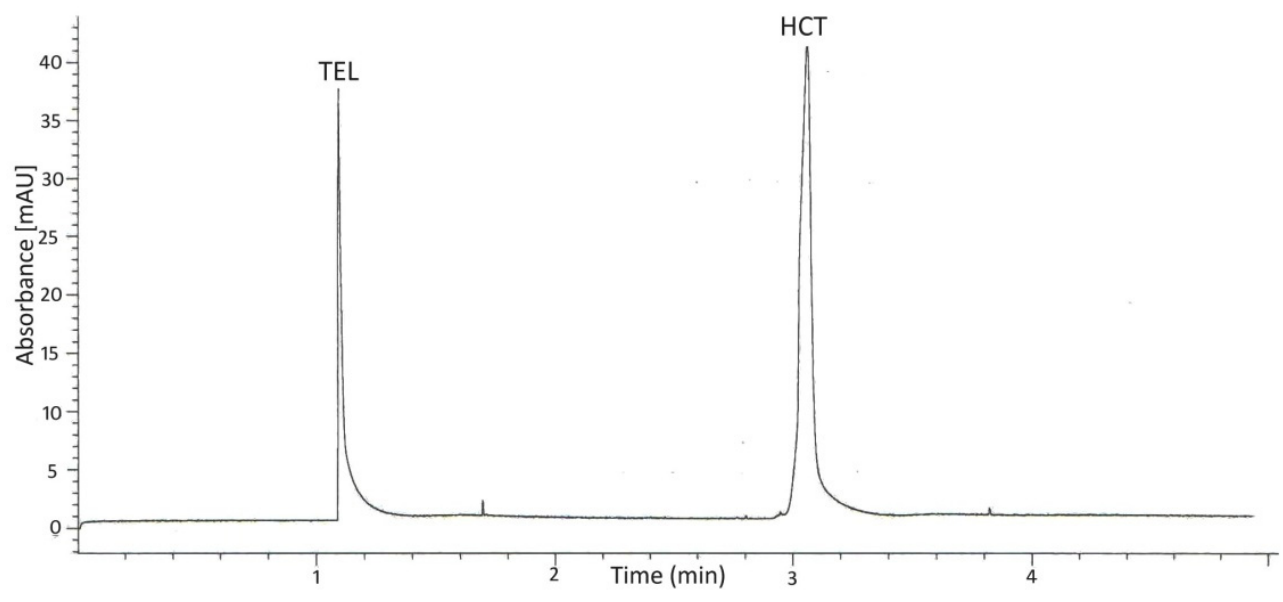

Figure 2. Simultaneous separation of TEL and HCT by ECZ (analytical conditions: $25 \mathrm{mM}$ phosphate, $\mathrm{pH} 2.50,+25 \mathrm{kV}, 25^{\circ} \mathrm{C}, 50 \mathrm{mbar} / 1 \mathrm{~s}$, UV $230 \mathrm{~nm}$ )

In the case of MEKC method, the optimal analytical conditions were: $25 \mathrm{mM}$ borate buffer, $25 \mathrm{mM}$ SDS, pH 9.50, applied voltage $+25 \mathrm{kV}$, capillary temperature $25{ }^{\circ} \mathrm{C}$, injection parameters $50 \mathrm{mbar} / 1 \mathrm{sec}$., UV detection wavelength $230 \mathrm{~nm}$. Using optimized condition, we obtained the separation of the two analytes, also in approximately 3 minutes, but the order of migration changed as HCT was followed by TEL (Figure 3).

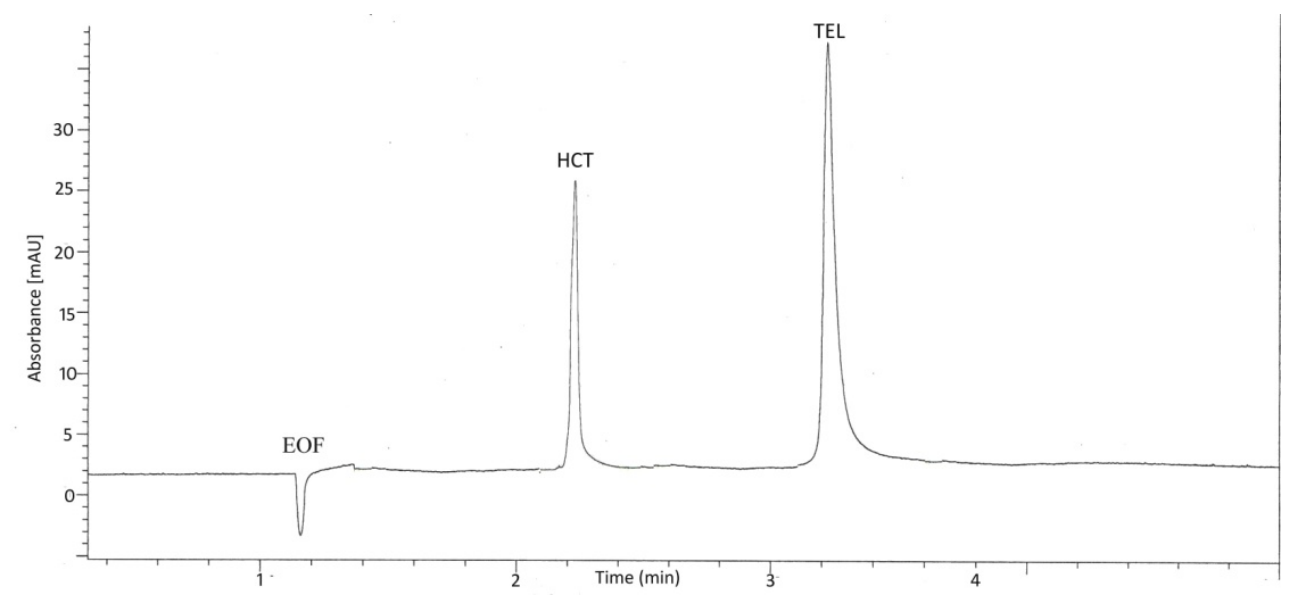

Figure 3. Simultaneous separation of TEL and HCT by MEKC (analytical conditions: $25 \mathrm{mM}$ borate, $25 \mathrm{mM}$ SDS, pH 9.50, $+25 \mathrm{kV}, 25^{\circ} \mathrm{C}, 50 \mathrm{mbar} / 1 \mathrm{~s}, \mathrm{UV} 230 \mathrm{~nm}$ ) 


\section{Analytical performances}

The analytical parameters of both CE methods were verified regarding precision, linearity, accuracy and robustness.

In order to evaluate intra-day and inter-day precision three concentration levels $\left(0.025,0.050,0.100 \mathrm{mg} \mathrm{mL}^{-1}\right)$ of standard solutions were used; the results were evaluated taking in consideration the peak areas. The intra-day determination was performed by analysing six replicates on the same day; while the inter-day determination was conducted over three consecutive days. The results are presented in Table 1.

Table 1. Intra- and inter-day precision for the simultaneous determination of TEL and HCT

\begin{tabular}{ccccc}
\hline Analyte $(\boldsymbol{\mu g} / \mathbf{m L})$ & \multicolumn{4}{c}{ RSD (\%) } \\
\cline { 2 - 5 } & \multicolumn{3}{c}{ CZE } & MEKC \\
\cline { 2 - 5 } & TEL & HCT & TEL \\
\hline $\begin{array}{c}\text { Intra-day precision } \\
\text { ( } \mathbf{n = 6 )}\end{array}$ & & & & \\
0.025 & 0.14 & 0.67 & 0.10 & 0.12 \\
0.050 & 0.18 & 0.83 & 0.18 & 0.21 \\
0.100 & 0.36 & 0.98 & 0.24 & 0.25 \\
Inter-day precision & & & & \\
( $\mathbf{n = 1 8 )}$ & & & & \\
0.025 & 0.52 & 0.77 & 0.28 & 0.33 \\
0.050 & 0.50 & 0.88 & 0.57 & 0.59 \\
0.100 & 0.95 & 1.12 & 0.80 & 0.82 \\
\hline
\end{tabular}

In order to evaluate linearity, stock solution at six concentration levels and three replicates per concentration were measured. The calibration curves were linear in the studied range $\left(0.010-0.500 \mathrm{mg} \mathrm{mL}^{-1}\right.$ for both TEL and HCT) with correlation coefficients above 0.99 (Table 2). Regression analysis data for the calibration curves were calculated using the peak areas.

Table 2. Linearity parameters for the simultaneous determination of TEL and HCT

\begin{tabular}{ccccc}
\hline Analytes & \multicolumn{2}{c}{ CZE } & \multicolumn{2}{c}{ MEKC } \\
\cline { 2 - 5 } & $\begin{array}{c}\text { Regression } \\
\text { equation }\end{array}$ & $\begin{array}{c}\text { Correlation } \\
\text { coefficient }\end{array}$ & $\begin{array}{c}\text { Regression } \\
\text { equation }\end{array}$ & $\begin{array}{c}\text { Correlation } \\
\text { coefficient }\end{array}$ \\
\cline { 2 - 6 } TEL & $y=167.5 x-1,483$ & 0.996 & $y=218.4 x+1.039$ & 0.991 \\
HCT & $y=234.4 x+4.191$ & 0.991 & $y=166.4 x+4.714$ & 0.991 \\
\hline
\end{tabular}


The approach based on the standard deviation of the response and the slope of the calibration plots was used to determine detection (LOD) and quantification limits (LOQ). LOD and LOQ values were estimated as [(standard deviation of the response)/ (slope of the regression equation)] by multiplying with 3.3 and 10 respectively. The values obtained are presented in Table 3.

Table 3. Sensitivity data for the simultaneous determination of INH and RIF

\begin{tabular}{ccccc}
\hline Analytes & \multicolumn{2}{c}{ CZE } & \multicolumn{2}{c}{ MEKC } \\
\cline { 2 - 5 } & LOD $(\mathrm{mg} / \mathrm{mL})$ & LOQ $(\mathrm{mg} / \mathrm{mL})$ & LOD $(\mathrm{mg} / \mathrm{mL})$ & LOQ $(\mathrm{mg} / \mathrm{mL})$ \\
\cline { 2 - 5 } TEL & 0.017 & 0.051 & 0.036 & 0.108 \\
HCT & 0.008 & 0.024 & 0.062 & 0.185 \\
\hline
\end{tabular}

To demonstrate the robustness of the method, minor changes in the experimental conditions have been made; as $\mathrm{pH}$ of the buffer was varied in the range $\pm 0.25 \mathrm{pH}$ units, separation temperature in the range $\pm 2{ }^{\circ} \mathrm{C}$ while applied voltage in the range $\pm 2 \mathrm{kV}$. None of the modifications caused significant changes in the resolution between the substances with RSD (\%) for migration times and peak areas under $2 \%$.

The accuracy study was performed by weighing an appropriate amount of Micardis Plus $80 / 25 \mathrm{mg}$ tablet powder and spiking it with known amount of standard compounds $\left(0.050,0.100,0.150 \mathrm{mg} \mathrm{mL}^{-1}\right)$; the resulting mixtures were analysed in triplicates and the results obtained were compared with the expected results. Accuracy values ranged from $98.32 \%$ to $101.75 \%$ for TEL and 96.26 and $100.42 \%$ for HCT in the ECZ method and from $99.12 \%$ to $102.11 \%$ for TEL and $99.04 \%$ to $102.36 \%$ for HCT in the MEKC method.

\section{Analysis of pharmaceutical formulations}

The optimized method was successfully applied for the simultaneous determination of TEL and HCT in the co-formulated original tablet, Micardis Plus $80 / 25 \mathrm{mg}$. Satisfactory results were obtained for each compound as the found amounts were in good agreement with label claims (Table 4).

Table 4. Assay results of TEL and HCT determination in pharmaceutical formulations

\begin{tabular}{ccccccc}
\hline Method & \multicolumn{2}{c}{ Declared amount $(\mathbf{m g})$} & \multicolumn{2}{c}{ Found amount $(\mathbf{m g})$} & \multicolumn{2}{c}{ RSD (\%) } \\
\cline { 2 - 7 } & TEL & HCT & TEL & HCT & TEL & HCT \\
\cline { 2 - 7 } CZE & 80 & 25 & 79.2 & 23.9 & 0.45 & 0.67 \\
MEKC & 80 & 25 & 79.4 & 24.2 & 0.75 & 0.82 \\
\hline
\end{tabular}




\section{CONCLUSIONS}

The usefulness of CZE and MEKC for the quantitative determination of these compounds in their pharmaceutical formulations was investigated, and the most important parameters for quantitative analysis were validated. The results presented above demonstrate that CE represents a good alternative for the simultaneous determination of TEL and HCT.

In CZE, at an acidic pH both analytes are positively charged, and the migration order can be explained by the differences between the own electrohphoretic mobilities of the analytes

In MEKC, both TEL and HCT are negatively charged and migrates after the EOF; the order of migration will depend on the individual partitioning equilibrium of the two analytes between the micellar and the aqueous phase. The greater percentage of analyte is distributed into the micelle, the slower it will migrate.

High separation efficiency and minimization of use of solvents and analytes are the inherent advantages features of both CE methods.

Both the CZE and the MEKC methods can be applied successfully to the qualitative and the quantitative determination of the studied compounds in fixed-dose pharmaceutical formulations.

\section{EXPERIMENTAL SECTION}

\section{Chemicals and Reagents}

Telmisartan and hydrochlorothiazide of pharmaceutical grade were obtained from RA Chem Pharma Limited (Hyderabad, India). Phosphoric acid was purchased from Chimopar (București, Romania), methanol and sodium hydroxide from Lach-Ner (Neratovice, Czech Republic), dihydrogen sodium phosphate, dissodium hydrogen phosphate, sodium tetraborate and sodium dodecyl sulfate from Merck (Darmstadt, Germany). All reagents were of analytical grade. Deionised water was purified by a Milli-Q purification system (Millipore, Bedford, USA). The pharmaceutical dosage forms used in this study was Micardis Plus (Boehringer Ingelheim, Germany) containing a TEL/HCT ratio of $80 / 25 \mathrm{mg}$.

\section{Instrumentation}

Separations were conducted on a Agilent 1600 CE system (Agilent Technologies, Waldbronn, Germany) equipped with a DAD detector. Uncoated fused-silica capillary of $38 \mathrm{~cm}$ length (30 cm effective legth) x $50 \mu \mathrm{m}$ ID (Agilent Technologies, Waldbronn, Germany) was used. Data acquisition was performed using ChemStation software (Agilent Technologies, Waldbronn, Germany). 


\section{Electrophoretic conditions}

The new capillary was conditioned by flushing with $0.1 \mathrm{~N} \mathrm{NaOH}$ and water each for 30 minutes. Between injections the capillary was preconditioned for 1 minute with $0.1 \mathrm{~N} \mathrm{NaOH}$, water and 2 minutes with the BGE. Samples and standards were injected hydrodynamically at the anodic end of the capillary. Detection was performed at 210, 230 and $270 \mathrm{~nm}$, and full spectra of the analytes were also stored to facilitate peak identification.

BGE solutions were prepared dissolving the appropriate amount of buffer constituents in ultrapure water and adjusting the $\mathrm{pH}$ if necessary with $1 \mathrm{M} \mathrm{H}_{3} \mathrm{PO}_{4}$ or $1 \mathrm{M} \mathrm{NaOH}$ solutions. Both $\mathrm{BGE}$ and sample solutions were filtered through a $0.45 \mu \mathrm{m}$ pore size membrane filter and sonicated in an ultrasonic bath for 5 minutes prior to use.

The stock solution of HCT was prepared in methanol in a concentration of $1 \mathrm{mg} / \mathrm{mL}$. The stock solution was prepared by dissolving $10 \mathrm{mg}$ TEL in in 1 $\mathrm{mL} 0.1 \mathrm{~N} \mathrm{HCl}$ and then diluting it to $10 \mathrm{~mL}$ with methanol.

\section{Preparation of pharmaceutical samples}

Twenty Micardis Plus $80 / 25 \mathrm{mg}$ tablets from the same batch were weighed accurately, average weight was calculated, the tablets were finely powdered in a mortar into a homogenous powder; an amount of powder equivalent to the weight of one tablet was dissolved in $100 \mathrm{~mL}$ methanol by sonication for 5 minutes with intermittent shaking. The solution was filtered through a $0.45 \mu \mathrm{m}$ syringe, centrifuged at $3500 \mathrm{rpm}$ for 10 minutes and diluted with methanol to the appropriate concentration. The same procedure was applied as in the separation from standard solutions.

\section{ACKNOWLEDGMENTS}

This research did not receive any specific grant from funding agencies in the public, commercial, or not-for-profit sectors.

\section{REFERENCES}

1. A. Salahuddin, M. Mustaq, B.J. Materson, Journal of American Society of Hypertension, 2013, 7, 401.

2. A.H. Gradman, J.N. Basile, B.L. Carter, G.L. Bakris, Journal of American Society of Hypertension, 2010, 4, 42. 
3. X. Wan, P. Ma, X. Zhang, Asian Journal of Pharmaceutical Sciences, 2014, 9, 1.

4. S.E. Kjeldsen, I. Os, A. Hojeggen, K. Beckey, G.W. Gleim, S. Oparil, American Journal of Cardiovascular Drugs, 2005, 5, 17.

5. M. Maillard, M. Burnier, Expert Review of Cardiovascular Therapy, 2005, 3, 375.

6. S. El Deeb, H. Watzig, D. Abd El-Hady, C. Sanger-van der Griend, G.K. Scriba, Electrophoresis, 2016, 37, 1591.

7. L.I. Bebawy, S.S. Abbas, L.A. Fattah, H.H. Refaat, II Farmaco, 2005, 60, 859.

8. R. Gangola, S. Kaushik, P. Sharma, Journal of Applied Pharmaceutical Sciences, 2011, 1, 46.

9. R.S. Bathe, A.M. Tamboli, M.J. Jamadar, N.I. Khan, J.Y. Manure, International Journal of Advances in Pharmaceutical Analysis, 2014, 4, 18.

10. I. Samala, Bulletin of Faculty of Pharmacy, Cairo University, 2011, 49, 19.

11. L.R. Bhat, R.K. Godge, A.T. Vora, M.C. Damle, Journal of Liquid Chromatograpgy \& Related Technologies, 2007, 30, 3059.

12. S.B. Wankhede, M.R. Tajne, K.R. Gupta, S.G. Wadodkar, Indian Journal of Pharmaceutical Sciences, 2007, 69, 298-300.

13. H.M. Hafez, A.A. Elshanawane, L.M. Abdelaziz, M. M. Kamal, Iranian Journal of Pharmaceutical Research, 2013, 12, 635.

14. S. Hillaert, W. van de Bossche, Journal of Pharmaceutical and Biomedical Analysis, 2003, 31, 329.

15. W. Wienen, M. Entzeroth, J.C.A. van Meel, J. Stangier, U. Busch, T. Ebner, J. Schmid, H. Lehmann, K. Matzek, J. Kempthorne-Rawson, V. Gladigau, N.H. Hauel, Cardiovascular Drug Reviews, 2000, 18, 127.

16. G.L. Plosker, W. B. White, Drugs, 2008, 68, 1877. 